Wilfrid Laurier University

Scholars Commons @ Laurier

Kinesiology and Physical Education Faculty

Publications

Kinesiology and Physical Education

2003

\title{
The Relationship Between Task Cohesion and Competitive State Anxiety
}

Mark A. Eys

Wilfrid Laurier University, meys@wlu.ca

James Hardy

University of Western Ontario

Albert V. Carron

University of Western Ontario

Mark R. Beauchamp

University of Leeds

Follow this and additional works at: https://scholars.wlu.ca/kppe_faculty

\section{Recommended Citation}

Eys, Mark A.; Hardy, James; Carron, Albert V.; and Beauchamp, Mark R., "The Relationship Between Task Cohesion and Competitive State Anxiety" (2003). Kinesiology and Physical Education Faculty Publications. 20.

https://scholars.wlu.ca/kppe_faculty/20

This Article is brought to you for free and open access by the Kinesiology and Physical Education at Scholars Commons @ Laurier. It has been accepted for inclusion in Kinesiology and Physical Education Faculty Publications by an authorized administrator of Scholars Commons @ Laurier. For more information, please contact scholarscommons@wlu.ca. 


\title{
The Relationship Between Task Cohesion and Competitive State Anxiety
}

\author{
Mark A. Eys, James Hardy, Albert V. Carron \\ University of Western Ontario
}

Mark R. Beauchamp

University of Leeds

\begin{abstract}
The general purpose of the present study was to determine if perceptions of team cohesion are related to the interpretation athletes attach to their precompetition anxiety. Specifically examined was the association between athlete perceptions of task cohesiveness (Individual Attractions to the GroupTask, ATG-T, and Group Integration-Task, GI-T) and the degree to which perceptions of the intensity of precompetition anxiety symptoms (cognitive and somatic) were viewed as facilitative versus debilitative. Participants were athletes $(N=392)$ from the sports of soccer, rugby, and field hockey. Each athlete completed the Group Environment Questionnaire (Carron, Widmeyer, \& Brawley, 1985) after a practice session. A directionally modified version of the Competitive State Anxiety Inventory-2 (Martens, Burton, Vealey, Bump, \& Smith, 1990) was completed just prior to a competition. Results showed that athletes who perceived their cognitive anxiety as facilitative had higher perceptions of both ATG-T and GI-T, $\chi^{2}(2, N=260)=8.96, p<.05$, than athletes who perceived their cognitive anxiety as debilitative. Also, athletes who perceived their somatic anxiety as facilitative had higher perceptions of GI-T, $\chi^{2}(2, N=249)=5.85, p<.05$.
\end{abstract}

Key Words: group dynamics, facilitators, debilitators

Prapavessis and Carron (1996) suggested that "improving the dynamics of the team could enhance the psychological state of the individual" (p. 72). Although applied research into developing a sense of "team" has been limited in sport psychology beyond anecdotal evidence (Carron \& Hausenblas, 1998), a major aspect of team dynamics that has been studied is team cohesion.

Cohesion has been constitutively defined as "a dynamic process which is reflected in the tendency for a group to stick together and remain united in the pursuit of its instrumental objectives and/or for the satisfaction of member affective needs" (Carron, Brawley, \& Widmeyer, 1998, p. 213). Carron, Widmeyer, and

M.A. Eys, J. Hardy, and A.V. Carron are with the School of Kinesiology, University of Western Ontario, London, ON, N6A 3K7 Canada. M.R. Beauchamp is with the Institute of Sport \& Exercise Sciences, University of Leed, Leeds, LS2 9JT, U.K. 
Brawley (1985) developed an operational definition of cohesion that included individual and group dimensions of both task and social cohesion. Thus, four dimensions emerged in their conceptualization: Individual Attraction to the Group-Task (ATG-T), Individual Attraction to the Group-Social (ATG-S), Group IntegrationTask (GI-T), and Group Integration-Social (GI-S).

Not surprisingly, the concept of cohesion has been empirically linked with a number of group and individual variables. Examples of group variables include a positive relationship with team performance (Carron, Bray, \& Eys, 2002), collective efficacy (Paskevich, 1995, Study 3), and group norms (Prapavessis \& Carron, 1997). Among the individual variables found to be associated with cohesion is competitive state anxiety (Prapavessis \& Carron, 1996). In sport, competitive state anxiety (A-state) is thought to derive largely from feelings of stress that arise from perceptions about the importance of the situation and uncertainty about the outcome (see Burton, 1998). Jones and Hanton (2001, p. 387) defined anxiety as "a negative cognitive and perceived physiological response to uncertain appraisals of coping with stressful demands." As implied in this definition, A-state is considered to be multidimensional, manifesting itself in three forms: somatic anxiety (e.g., increased heart rate, sweating), cognitive anxiety (e.g., self-doubt, concerns about losing), and self-confidence (e.g., at ease, secure) (Martens, Burton, Vealey, Bump, \& Smith, 1990). All three manifestations of anxiety are considered to have different antecedents and consequences (Burton, 1998).

As indicated above, the relationship between cohesion and competitive state anxiety has previously been explored. Prapavessis and Carron (1996) assessed the perceptions of cohesiveness of 110 "A-grade athletes" (p. 66) with a mean age of 23.40 years from a cross-section of sports (rugby, basketball, hockey, and soccer) at a midweek practice and then assessed the intensity of cognitive anxiety symptoms, somatic anxiety symptoms, and self-confidence 15 minutes prior to the next competition. They found that athletes who expressed greater cohesion manifested as Individual Attractions to Group-Task (ATG-T) had a lower level of precompetition cognitive anxiety.

One caveat is offered, however: in all situations-social, business, academia, and sport - anxiety has been traditionally viewed as a negative construct that is detrimental to performance. Recent research and theoretical discussions by Jones and colleagues (Jones \& Swain, 1992; Jones, Swain, \& Hardy, 1993) have served to point out that while existing measures of precompetition anxiety symptoms (e.g., Competitive State Anxiety Inventory-2; Martens et al., 1990) tap the intensity of symptoms, they do not assess the direction or interpretation the individual attaches to those symptoms.

Thus, for example, two participants may have highly intense somatic symptoms prior to a sport competition (e.g., "My palms are sweaty"). For one competitor, the manifestations of these symptoms may be interpreted in a negative way because they reflect unease and uncertainty (e.g., "My anxiety reflects my unease"). This situation is referred to as debilitative anxiety. For another, however, the symptom manifestations may be interpreted in a positive way because they reflect a degree of readiness necessary for success (e.g., "I'm ready to begin competing"). This positive interpretation is referred to as facilitative anxiety.

Jones and Hanton (2001) summarized the current debate regarding the terminology used in the literature on anxiety insofar as the relationship between precompetitive feeling states and directional anxiety interpretations is concerned. 
They noted that the term facilitative anxiety may represent a potential contradiction. As was discussed above, anxiety typically carries a negative connotation. Therefore, to describe an individual as experiencing "facilitative anxiety" could be an oxymoron. Those who interpret certain cognitive (e.g., concern) or somatic (e.g., sweaty palms) symptoms as being positive may not be experiencing anxiety at all, but rather another phenomenon such as excitement or a sense of challenge.

In the present study, the distinction between negative and positive interpretations has been acknowledged. In other words, when individuals interpreted cognitive and somatic symptoms as being negative, the responses have been referred to as debilitative interpretations of symptoms of anxiety. If individuals interpreted the described symptoms as being positive, however, then the responses have been labeled as facilitative interpretations of symptoms, not facilitative anxiety.

Research has provided support for the need to distinguish between the intensity and direction dimensions when measuring precompetition cognitive and somatic symptoms. Jones and Hanton (1996), in a study of the relationship between expectancies about goal attainment and both the intensity and direction of symptoms, reported that nearly half of their sample of elite athletes interpreted the intensity of their symptoms as facilitative (helpful) to performance. Also, Jones and Swain (1995) found no differences in the intensity levels of precompetition symptoms in elite and non-elite athletes, but the former interpreted their cognitive and somatic symptoms as being more facilitative to performance than did the latter. Jones and Hanton (2001) suggested, "it would appear that not only is there value in examining the interpretation of responses, but also that 'direction' appears to be more sensitive in distinguishing between individual difference groups when compared with the intensity of responses" (p. 386).

Therefore, the general purpose of our study was to extend the work of Prapavessis and Carron (1996) by determining how perceptions of team cohesion are related to the interpretation that athletes attach to precompetition cognitive and somatic symptoms. Specifically, we examined the association between athlete perceptions of task cohesiveness (Individual Attractions to the Group-Task, ATG-T, and Group Integration-Task, GI-T) and the degree to which perceptions of the presence of precompetition cognitive and somatic symptoms are viewed as facilitative or debilitative for performance.

Only the task dimensions of cohesion (instead of also including the social dimensions) were assessed, for two reasons. First, Prapavessis and Carron (1996) only found a relationship between the intensity of precompetition symptoms and the cohesion dimension ATG-T. Second, they also investigated the possible mediational effect of perceptions of the psychological costs and benefits of team cohesion within the cohesion/anxiety relationship. Prapavessis and Carron found that individuals who perceived high levels of cohesion were more likely to indicate that the pressure to carry out responsibilities and satisfy the expectations of others was reduced and therefore they experienced less anxiety. The pressure to carry out responsibilities and satisfy the expectations of others can be assumed to be taskoriented activities.

The Prapavessis and Carron proposals (and findings relative to cognitive anxiety, somatic anxiety, ATG-T, and GI-T) were considered to have relevance for the present study. Thus, it was hypothesized that there would be a positive relationship between the tendency to perceive cognitive symptoms as more facilita- 
tive (vs. more debilitative) and the tendency to perceive greater ATG-T. It was also hypothesized that there would be no relationship between the interpretation of precompetitive cognitive symptoms and GI-T, and the interpretation of precompetitive somatic symptoms and either ATG-T or GI-T.

\section{Method}

\section{Participants and Measures}

Participants in the study were 276 male and 116 female athletes from a variety of interactive sports who competed at the intercollegiate, club, or competitive school level. These included soccer $(n=97)$, rugby $(n=170)$, and field hockey $(n$ $=125$ ). There was heterogeneity in competitive level within all three sports. The mean age of the athletes was 17.12 years $(S D=3.76)$.

Task Cohesion. Task cohesion was assessed using the Group Environment Questionnaire (GEQ; Carron et al., 1985). The GEQ consists of 18 items that assess four dimensions of cohesion: Group Integration-Task (GI-T; 5 items), which measures the individual's perception about the degree of unity in the team as a collective around its goals and objectives; Group Integration-Social (GI-S; 4 items), which measures the individual's perception about the degree of social unity in the team as a collective; Individual Attractions to the Group-Task (ATG-T; 4 items), which measures the individual's perception about his or her own involvement in task oriented aspects of the group; and Individual Attractions to the Group-Social (ATG-S; 5 items), which measures the individual's perception about his or her own involvement in social aspects of the group. As noted above, only the two task oriented scales (ATG-T and GI-T) were used in the present study.

Participants were instructed to indicate the extent to which they agreed with each statement on the questionnaire. A 9-point Likert scale was used, anchored at the extremes by 1 ("strongly disagree") and 9 ("strongly agree"). Thus, higher scores reflected stronger perceptions of cohesiveness. Internal consistency values computed with the data secured for the present study showed acceptable values for both scales: ATG-T, $\alpha=.66$; GI-T, $\alpha=.71$.

Precompetition Symptoms. Precompetition symptoms were assessed using a modified version of the Competitive State Anxiety Inventory-2 (CSAI-2; Martens et al., 1990). The CSAI-2 has three subscales that assess cognitive symptoms, somatic symptoms, and self-confidence. Only the Cognitive and Somatic subscales were used for the present study. Each subscale contains 9 items. To determine the intensity of symptoms, participants responded to the 18 questions on a 4-point Likert scale that ranged from 0 ("not at all") to 3 ("very much so").

A directional scale for each question was also included, consistent with the method employed by Jones and Swain (1992). That is, each participant was asked to rate the extent to which he or she felt that each symptom (i.e., the 18 items) was either debilitative or facilitative to his or her performance. Responses were obtained on a 7-point Likert scale that started at -3 ("very debilitative"), included a 0 ("unimportant"), and continued on to +3 ("very facilitative").

Adequate alpha values were obtained for all intensity (cognitive, $\alpha=.74$; somatic, $\alpha=.83$ ) and direction (cognitive, $\alpha=.79$; somatic, $\alpha=.85$ ) components of the modified CSAI-2. 


\section{Procedure}

Recruitment and contact of the participants was carried out following an initial meeting with the head coach of each team. After securing the coach's approval, we attended a practice session in midweek to explain the nature of the study. At that time the athletes read the letter of information and completed the consent form and the Group Environment Questionnaire. Participants under the age of 18 years completed the GEQ at the next practice session after obtaining legal guardian permission. The modified version of the CSAI-2 was completed 30-45 minutes prior to the next regular season game. We assured the participants of confidentiality from coaches and other players, as well as access to the general results upon completion of the study.

\section{Data Analysis}

In analyzing the data, we made two departures from the typical method of statistical examination in the literature on competitive state anxiety. First we determined a composite score for each individual on the interpretation of cognitive and somatic symptoms by summing only those items the athlete indicated he or she was experiencing; if an athlete responded "not at all (0)" to an item, the interpretation-facilitative, unimportant, debilitative-was not considered. That is, those symptoms the individual indicated he/she did not experience were removed from the analysis of the interpretation of cognitive and somatic symptoms. The result was a cognitive and somatic score ranging from -27 (i.e., had a symptom response on all 9 items within the dimension of some intensity, and interpreted all of them as debilitative to performance) to +27 (i.e., had a symptom response on all 9 items within the dimension of some intensity and interpreted all of them as facilitative to performance).

This procedure was followed to ensure that the question of interpretation was consistent on each item for each participant. That is, to ask an individual to interpret the absence of a symptom is different from asking him or her to interpret a symptom that exists to some degree; these qualitatively different responses should not be combined. Implications of this important methodological departure are elaborated upon in the Discussion section.

The second departure from typical analyses was that two groups of athletes were created. Those with scores at one extreme tertile of the distribution formed what is referred to hereafter as the Debilitative Group (composed of athletes who experienced symptoms of some intensity but the sum of their interpretations of these symptoms was negative). Athletes with scores at the other extreme tertile of the distribution formed what is referred to hereafter as the Facilitative Group. The middle third of the distribution was not used in any subsequent analyses.

It should be noted that categorization into the Debilitative and Facilitative Groups was carried out independently for cognitive and somatic anxiety. Given that Prapavessis and Carron (1996) found only a mild relationship between cohesion (ATG-T) and the intensity of cognitive symptoms, a tertile split (extreme groups) analysis was employed to enhance the ability to distinguish between individuals who tended to view their symptoms as being more facilitative versus those who viewed their symptoms as debilitative. This extreme-group approach with continuous data has been used in a number of studies. For example, Rascle, Cou- 
Table 1 Means and Standard Deviations for Cognitive and Somatic Interpretation Groups

\begin{tabular}{cccccccccccccc}
\hline & \multicolumn{1}{c}{ ATG-T } & \multicolumn{1}{c}{ GI-T } & \multicolumn{1}{c}{ CogInt } & \multicolumn{1}{c}{ SomInt } & \multicolumn{2}{c}{ CogDir } & \multicolumn{2}{c}{ SomDir } \\
& $M$ & $S D$ & $M$ & $S D$ & $M$ & $S D$ & $M$ & $S D$ & $M$ & $S D$ & $M$ & $S D$ \\
\hline & & & & & & & & & & & & \\
Cognitive & & & & & & & & & & & & & \\
Facil. & 7.01 & 1.47 & 6.35 & 1.43 & 1.20 & 0.48 & 0.60 & 0.47 & 1.35 & 0.62 & 0.96 & 0.99 \\
Debil. & 6.53 & 1.78 & 5.82 & 1.51 & 1.14 & 0.60 & 0.79 & 0.57 & -.47 & 0.80 & 0.07 & 1.03 \\
Somatic & & & & & & & & & & & & & \\
Facil. & 6.91 & 1.62 & 6.36 & 1.46 & 1.15 & 0.49 & 0.72 & 0.41 & 0.80 & 0.95 & 1.06 & 0.81 \\
Debil. & 6.75 & 1.76 & 5.90 & 1.52 & 1.29 & 0.62 & 0.95 & 0.57 & -.11 & 1.00 & -.28 & 0.89 \\
& & & & & & & & & & & & \\
\hline
\end{tabular}

Note: ATG-T = Individual Attraction to Group-Task; GI-T = Group Integration-Task; CogInt $=$ Cognitive Intensity; SomInt $=$ Somatic Intensity; CogDir $=$ Cognitive Direction (interpretation); SomDir = Somatic Direction (interpretation); Facil. = Facilitator Group; Debil. $=$ Debilitator Group.

lomb, and Pfister (1998) used an extreme-group approach (quartile split) to determine whether high-ego-oriented athletes differed in instrumental and hostile aggression than athletes they classified as low-ego-oriented.

\section{Results}

The means and standard deviations for all relevant variables are listed in Table 1. Also, bivariate correlations between the primary variables are presented in Table 2. To ensure that the use of extreme groups (upper and lower tertile) actually involved a comparison of different groups, we conducted an analysis of variance (ANOVA) with group membership as the independent variable and the interpretational response as the dependent variable. For both the cognitive, $F(1$, $258)=214.45, p<.01$, and somatic, $F(1,247)=144.18, p<.01$, domains, facilitators interpreted their symptoms more positively than did debilitators.

Two direct discriminant function analyses were conducted. Debilitative and facilitative groups were formed on the basis of cognitive responses in the first discriminant function analysis, and on the basis of somatic responses in the second. For both discriminant function analyses, the two measures of cohesion (ATGT and GI-T) represented the independent variables while interpretation of symptoms (debilitative group and facilitative group) represented the dependent variable. Prior to conducting the analyses for both cognitive and somatic anxiety responses, we examined both groups for outliers and found no extreme outliers in the present study.

Relative to the analysis carried out on the basis of cognitive anxiety responses, a significant difference was obtained between the facilitative group and the debilitative group, $\chi^{2}(2, N=260)=8.96, p<.05$. The discriminant function analy- 
Table 2 Bivariate Correlations Among the Variables of Interest

\begin{tabular}{|c|c|c|c|c|c|c|c|}
\hline & GI-T & CogInt & SomInt & CogDir & SomDir & CogComp & SomComp \\
\hline ATG-T & $.576^{* *}$ & $-.106^{*}$ & -.068 & .063 & .038 & $.107 *$ & .099 \\
\hline GI-T & & $-.125^{*}$ & $-.118^{*}$ & .071 & .036 & $.113^{*}$ & $.115^{*}$ \\
\hline CogInt & & & $.573 * *$ & $-.127^{*}$ & $-.149 * *$ & .022 & -.075 \\
\hline SomInt & & & & $-.226 * *$ & $-.268 * *$ & $-.158 * *$ & -.067 \\
\hline CogDir & & & & & $.672 * *$ & $.783 * *$ & $.383 * *$ \\
\hline SomDir & & & & & & $.349 * *$ & $.521 * *$ \\
\hline CogComp & & & & & & & $.461 * *$ \\
\hline
\end{tabular}

Note $:$ ATG-T = Individual Attraction to Group-Task; GI-T = Group Integration-Task; CogInt = Cognitive Intensity; SomInt = Somatic Intensity; CogDir = Cognitive Direction (interpretation); SomDir $=$ Somatic Direction (interpretation); CogComp $=$ Cognitive Composite score; SomComp = Somatic Composite score.

* Correlation is signif. at 0.05 level (2-tailed); ${ }^{*}$ Correlation is signif. at 0.01 level (2-tailed).

Table 3 Means and Standard Deviations of Cognitive Component of Competitive State Anxiety

\begin{tabular}{|c|c|c|c|c|c|c|c|c|}
\hline \multirow{2}{*}{$\begin{array}{l}\text { GEQ } \\
\text { scales }\end{array}$} & \multicolumn{2}{|c|}{ Facilitators } & \multicolumn{2}{|c|}{ Debilitators } & \multirow[b]{2}{*}{$F$} & \multirow[b]{2}{*}{$p$} & \multirow{2}{*}{$\begin{array}{l}\text { Stand. D.F. } \\
\text { coefficient }\end{array}$} & \multirow{2}{*}{$\begin{array}{l}\text { Structure } \\
\text { coefficien }\end{array}$} \\
\hline & $M$ & $S D$ & $M$ & $S D$ & & & & \\
\hline ATG-T & 7.01 & 1.47 & 6.53 & 1.78 & 5.86 & .016 & .391 & .800 \\
\hline GI-T & 6.35 & 1.43 & 5.83 & 1.51 & 8.20 & .005 & .726 & .946 \\
\hline
\end{tabular}

Note: $57.3 \%$ of original grouped cases correctly classified. ATG-T = Individual Attraction to Group-Task; GI-T = Group Integration-Task; Stand. D.F. coefficient $=$ Standardized Discriminant Function coefficient.

sis correctly classified $57.3 \%$ of the sample (see Table 3 ). One-way ANOVAs computed to compare the groups showed that the two groups of athletes differed significantly on perceptions of cohesion. Athletes in the facilitative group had higher perceptions of both ATG-T (which supported our hypothesis) and GI-T (which was contrary to our hypothesis), compared to athletes in the debilitative group.

Also contrary to our hypothesis, a significant difference was obtained between groups when group membership was based on somatic responses, $\chi^{2}(2, N=$ $249)=5.85, p<.05$. The discriminant function analysis correctly classified $59.0 \%$ of the sample (see Table 4). Again, one-way ANOVAs were computed on both cohesion measures to compare the facilitative and debilitative groups. Differences 
Table 4 Cohesion Means and Standard Deviations of the Somatic Component of Competitive State Anxiety

\begin{tabular}{|c|c|c|c|c|c|c|c|c|}
\hline \multirow{2}{*}{$\begin{array}{l}\text { GEQ } \\
\text { scales }\end{array}$} & \multicolumn{2}{|c|}{ Facilitators } & \multicolumn{2}{|c|}{ Debilitators } & \multirow[b]{2}{*}{$F$} & \multirow[b]{2}{*}{$p$} & \multirow{2}{*}{$\begin{array}{l}\text { Stand. D.F. } \\
\text { coefficient }\end{array}$} & \multirow{2}{*}{$\begin{array}{l}\text { Structure } \\
\text { coefficien }\end{array}$} \\
\hline & $M$ & $S D$ & $M$ & $S D$ & & & & \\
\hline ATG-T & 6.91 & 1.62 & 6.75 & 1.76 & .509 & .476 & -.389 & .293 \\
\hline GI-T & 6.36 & 1.46 & 5.90 & 1.52 & 5.350 & .022 & 1.174 & .949 \\
\hline
\end{tabular}

Note: $59.0 \%$ of original grouped cases correctly classified. ATG-T = Individual Attraction to Group-Task; GI-T = Group Integration-Task; Stand. D.F. coefficient $=$ Standardized Discriminant Function coefficient.

in means were only found for GI-T, with athletes in the facilitative group having stronger perceptions of cohesion than athletes in the debilitative group.

In order to more fully understand the relationship between team cohesion and the directional interpretation of symptoms, we set out to explain the discriminant functions generated by the two analyses. This involved examination of both full (structure coefficients) and partial (standardized discriminant functions coefficients) relationships of the variables to the weighted multivariate function. The results, summarized in Tables 3 and 4, showed that in both discriminant function analyses, GI-T contributed more to the discrimination between the facilitator and debilitator groups than did ATG-T. In fact, as Table 3 shows, with regard to the function generated to discriminate between facilitators and debilitators of somatic symptoms, ATG-T was not related strongly enough (i.e., structure coefficient <.30) to warrant its inclusion for interpretation of the discriminant function. In short, it seems that perceptions of GI-T are a more important predictor of the degree to which precompetition anxiety is viewed as facilitative or debilitative. However, the relationships we found, although significant, are weak. For both analyses (somatic and cognitive), the percent of athletes correctly classified did not reach $60 \%$, and thus the conclusions are limited.

\section{Discussion}

The purpose of the present study was to determine how the interpretation that athletes attach to symptoms of precompetition anxiety is related to perceptions of cohesion. Specifically, the association between athlete perceptions of task cohesiveness (ATG-T and GI-T) and the degree to which perceptions of precompetition anxiety symptoms are viewed as facilitative or debilitative was examined. The results showed that athletes who viewed their cognitive symptoms as facilitative also had more positive perceptions of both ATG-T and GI-T. Furthermore, athletes who viewed their somatic symptoms as facilitative had more positive perceptions of GI-T.

The benefits of belonging to a cohesive group have been well documented. These benefits include improved mood (Terry, Carron, Pink, et al., 2000), increased 
self-esteem (Julian, Bishop, \& Fielder, 1966), increased belief in the group's ability to withstand negative circumstances or disruption to the group (Brawley, Carron, \& Widmeyer, 1988), and a greater readiness of members to diffuse responsibility for failure throughout the group (Brawley, Carron, \& Widmeyer, 1987). Such benefits may account for why athletes who perceive higher levels of cohesion tend to view symptoms as being more facilitative. Again, it must be kept in mind that the association for both domains were somewhat weak based on the percent who were correctly classified. This was not unexpected, however, based on the results from Prapavessis and Carron (1996).

Research by Prapavessis and Carron (1996) showed a relationship between the intensity of precompetitive cognitive state anxiety and perceptions of cohesion manifested as ATG-T. Although the nature of the issue pursued by them and by us was similar, our results differed from those of Prapavessis and Carron in three important ways. First, Prapavessis and Carron found that only precompetition cognitive anxiety was related to cohesiveness; we found a relationship between both perceptions of cohesiveness and precompetition cognitive and somatic anxiety. Second, they found that only ATG-T was related to precompetition anxiety; we found that both GI-T and ATG-T were related to precompetition anxiety. In fact, our research showed that the state anxiety/cohesion relationship was stronger for GI-T. Finally, and most important, Prapavessis and Carron (only examined and) found a relationship between cohesion and the intensity of precompetition anxiety. Our research has shown that perceptions of cohesiveness may be associated with the interpretation attached to (i.e., the direction) the precompetition symptoms experienced by athletes.

The literature on precompetition anxiety is increasingly characterized by the tendency to take into account both its intensity and direction. Our work contained a methodological departure from previous studies that should be highlighted — and perhaps incorporated-into subsequent research on precompetition anxiety. Previous research has not taken into account the fact that some athletes may report a lack of intensity in various anxiety symptoms, such as the 18 items in the CSAI-2 used in the present study. Consequently, it is possible for an athlete to respond "not at all" to any or all 18 items and then go on to report that this is very facilitative to performance. However, an athlete reporting "not at all" is not experiencing the presence of the symptom but rather the absence of it. The presence versus absence of precompetition anxiety symptoms represents two conceptually different statespossibly with different antecedents and consequences.

Although the results were limited, the study may have implications for theory advancement. Our findings reinforce those of Prapavessis and Carron (1996) insofar as members of highly task cohesive teams are likely to experience competitive state anxiety differently than members of less cohesive teams. The findings suggest that the perception of cohesion is related to how individuals interpret their experience of anxiety symptoms. However, causation cannot be inferred from the results of the study. Therefore, future research should seek to utilize experimental designs to understand the impact of increased task cohesion upon the affective states of athletes, and the interpretation the athletes attach to those affective perceptions.

There are also practical implications of the results from the present study. Previous research has shown that personal variables, such as competitiveness (Jones 
\& Swain, 1992) and the expectancy about goal attainment (Jones \& Hanton, 1996), are related to the directional interpretation of state anxiety symptoms. The present study showed that the team environment is also related to athletes' interpretation of anxiety symptoms. As such, this lends support to Prapavessis and Carron's (1996) suggestion that even though most interventions utilized to enhance the psychological state of the athlete focus on personal variables, "improving the dynamics of the team could [also] enhance the psychological state of the individual" (p. 72).

In summary, the present study examined the relationship between cohesion and the interpretation of precompetition symptoms using composite scores, combining both intensity and directional perceptions of cognitive and somatic symptoms. A significant relationship was found between the two variables. Specifically, the more positive their perceptions of task cohesion, the more facilitative the athletes interpreted their state anxiety symptoms.

\section{References}

Brawley, L.R., Carron, A.V., \& Widmeyer, W.N. (1987). Assessing the cohesion of teams: Validity of the Group Environment Questionnaire. Journal of Sport Psychology, 9, 275-294.

Brawley, L.R., Carron, A.V., \& Widmeyer, W.N. (1988). Exploring the relationship between cohesion and group resistance to disruption. Journal of Sport \& Exercise Psychology, 10, 199-213.

Burton, D. (1998). Measuring competitive state anxiety. In J.L. Duda (Ed.), Advancements in sport and exercise psychology measurement (pp. 129-148). Morgantown, WV: Fitness Information Technology.

Carron, A.V., Brawley, L.R., \& Widmeyer, W.N. (1998). The measurement of cohesiveness in sport groups. In J.L. Duda (Ed.), Advancements in sport and exercise psychology measurement (pp. 213-226). Morgantown, WV: Fitness Information Technology.

Carron, A.V., Bray, S.R., \& Eys, M.A. (2002). Team cohesion and team success in sport. Journal of Sport Sciences, 20, 119-226.

Carron, A.V., \& Hausenblas, H.A. (1998). Group dynamics in sport (2nd ed.). Morgantown, WV: Fitness Information Technology.

Carron, A.V., Widmeyer, W.N., \& Brawley, L.R. (1985). The development of an instrument to assess cohesion in sport teams: The Group Environment Questionnaire. Journal of Sport Psychology, 7, 244-266.

Jones, G., \& Hanton, S. (1996). Interpretation of competitive anxiety symptoms and goal attainment expectancies. Journal of Sport \& Exercise Psychology, 18, 144-157.

Jones, G., \& Hanton, S. (2001). Pre-competitive feeling states and directional anxiety interpretations. Journal of Sports Sciences, 19, 385-395.

Jones, G., \& Swain, A. (1992). Intensity and direction as dimensions of competitive state anxiety and relationships with competitiveness. Perceptual and Motor Skills, 74, 467-472.

Jones, G., \& Swain, A. (1995). Predispositions to experience debilitative and facilitative anxiety in elite and nonelite performers. The Sport Psychologist, 9, 201-211.

Jones, G., Swain, A., \& Hardy, L. (1993). Intensity and direction dimensions of competitive state anxiety and relationships with performance. Journal of Sports Sciences, 11, $525-532$. 
Julian, J., Bishop, D., \& Fielder, F.E. (1966). Quasitherapeutic effects of intergroup competition. Journal of Personality and Social Psychology, 3, 321-327.

Martens, R., Burton, D., Vealey, R.S., Bump, L.A., \& Smith, D.E. (1990). The Competitive State Anxiety Inventory-2 (CSAI-2). In R. Martens, R.S. Vealey, \& D. Burton, Competitive anxiety in sport (pp. 117-190). Champaign, IL: Human Kinetics.

Paskevich, D.M. (1995). Conceptual and measurement factors of collective efficacy in its relationship to cohesion and performance outcome. Unpublished doctoral dissertation, University of Waterloo, Ontario.

Prapavessis, H., \& Carron, A.V. (1996). The effect of group cohesion on competitive state anxiety. Journal of Sport \& Exercise Psychology, 18, 64-74.

Prapavessis, H., \& Carron, A.V. (1997). The role of sacrifice in the dynamics of sports teams. Group Dynamics, 1, 231-240.

Rascle, O., Coulomb, G., \& Pfister, R. (1998). Aggression and goal orientations in handball: Influence of institutional sport context. Perceptual and Motor Skills, 86, 13471360.

Terry, P.C., Carron, A.V., Pink, M.J., Lane, A.M., Jones, G.J.W., \& Hall, M.P. (2000). Perceptions of group cohesion and mood in sport teams. Group Dynamics, 4, 244-253.

Manuscript submitted: May 17, 2001

Revision accepted: November 5, 2002 